\title{
Design and Experiment of a New Solar Automatic Tracking System
}

\author{
Lili Cheng ${ }^{1}$ and Bin Wang ${ }^{2}$ \\ ${ }^{1}$ Institute of Technology , Jilin University, 130012, Changchun, China \\ ${ }^{2}$ CRRC Qishuyan Institute Co.,Ltd, 213011, Changzhou, China
}

\begin{abstract}
A new type of solar photovoltaic power generation automatic tracking system was designed in this paper. First of all, based on the principle of dual-axes tracking and the law of the sun trajectory, a novel parallel solar tracking mechanism was devised. The mechanism of automatic tracking system uses 3-DOF parallel structure and can track the sun all-round. Secondly, the control algorithm is studied by the mathematical model of parallel tracking mechanism and proposed the tracking strategy of the photoelectric tracking and sun trajectory tracking. In the sunny day, the sunlight is detected by photoelectric sensor and with the photoelectric tracking mode. In the rainy days, according to the calculation of the solar altitude angles and azimuth, the automatic tracking is used the construction of the data base and look-up table to track sun by software control method. Finally, the generating capacity of automatic tracking system experiment is carried on research. The experimental results show that the designed parallel tracking mechanism has a good performance and stable operation, also can realize all-round automatic tracking. The power generation of the new automatic tracking system has more power than two axes tracking $14.1 \%$.
\end{abstract}

Keywords-automatic tracking; parallel mechanism; photoelectric tracking; sun trajectory tracking

\section{INTRODUCTION}

As an effective way to improve the utilization of solar energy, solar automatic tracking system has been paid attention to by the countries all over the world. Solar automatic tracking means that during the process of solar radiation, the solar panel's surface is always perpendicular to the solar rays and more solar radiation energy is obtained in a limited area [1,2]. The sun energy acceptance rate is different with another tracking method. At present, the tracking mechanism can be divided into single axis tracking, two axis tracking, polar axis tracking and parallel tracking, and so on [3]. Two axis tracking is the mainstream method at present. It can automatically track the sun in two directions of horizontal and pitch. It also can achieve larger power output. In recent years, the parallel mechanism has large stiffness, stable structure, high accuracy, and easy to realize real-time motion control, so it has been gradually applied to the solar automatic tracking. The typical 3-DOF parallel sphere tracking mechanism is proposed by Professor Zhang Shunxin in Hebei University of Technology [4]. The parallel spherical tracking mechanism has the characteristics of compact structure, high stiffness and large working space. It can achieve a comprehensive tracking and greatly efficiency of acceptance.

The existing tracking mechanism is driven directly by the motor. A heavier solar panel will be used a large motor drive which makes the power consumption of its own increase and the effective output power generation decrease $[5,6]$. In order to solve the problem of sun tracking mechanism, a novel solar automatic tracking system was designed this paper. The tracking system can reduce the power loss of its own and increase the output effective power generation. Therefore, it can improve the efficiency of tracking device and achieve the purpose of automatic tracking.

\section{Design Of New Solar Tracking MEchanism}

A. The Working Principle of a New Parallel Automatic Tracking Mechanism

The new solar tracking system mainly includes support mechanism, limiting mechanism, drive mechanism and parallel steering mechanism. The supporting mechanism is composed of a tripod support at the bottom, a triangular platform at the top and a universal joint fixed on it. The driving mechanism passes the torque to the gear through the stepping motor. Then the gear-rack passes are meshed through the steel wire which the position and the posture of the solar energy plate are changed arbitrarily. Three sets of limit rod in triangular platform and the solar panel tray are made up the limiting mechanism. The upper of three sets limit rod is connected a spherical hinge to the solar plate tray. The 3 spherical hinges are fixed on the solar panel tray and formed a triangle. The steering mechanism is composed of 3 spherical hinges in the solar plate tray edge of the 120 degrees angle and formed a triangle. The hinged thin steel wire is fixed on the rack by the guide wheel. The three-dimensional model is shown in Fig.1.

The working principle of the mechanism is that the stepping motor is powered on, then the thin steel wire connected with the gear is driven by a gear-rack mechanism, so the tray movement is influenced by the guide wheel. The coordinated movement of the three fine wire ropes makes the attitude of the solar panel change arbitrarily. The azimuth angle can reach 360 degrees, and the pitching angle also can reach 110 degrees. Therefore, the full range tracking can be realized. 


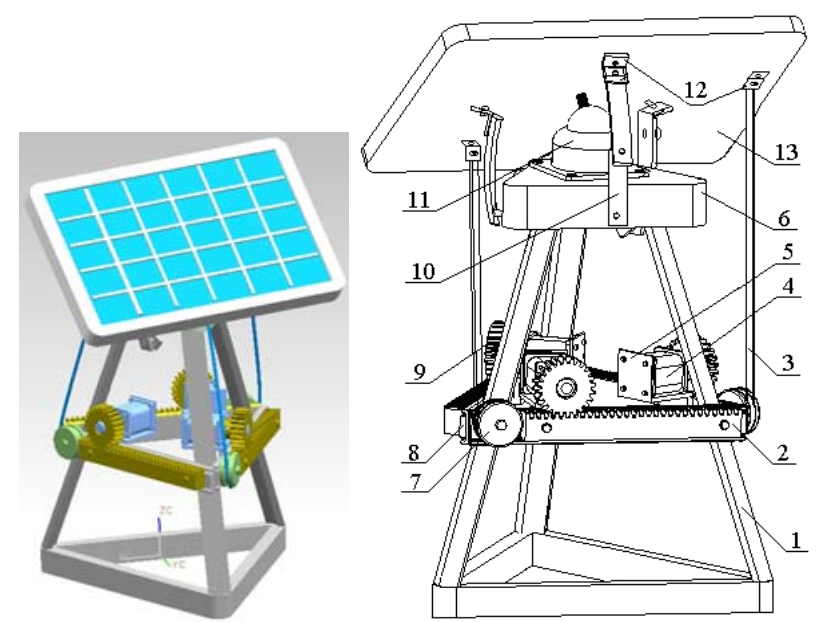

1.Triangle support 2.Rack 3.Thin steel wire 4.Stepping motor 5.Motor bracket 6.Triangular platform 7.Guide wheel 8.Guide rail 9.Gear 10.Limit connecting rod 11.Universal coupling 12.Spherical hinge 13 . Solar panel tray

\section{FIGURE I. THE NEW SUN TRACKING MECHANISM MODEL}

\section{B. Modeling of New Parallel Automatic Tracking Mechanism}

In order to facilitate the establishment of a mathematical model, the new parallel tracking mechanism was designed in this paper. The mechanism can be divided into fixed platform and moving platform. The platform is composed of three stepping motors, gear-rack and guide wheel. The moving platform is composed of solar panel tray and universal coupling. The 3 spherical hinges are arranged at the edge of the solar panel tray at 120 degrees intervals, and the three fixed points of the 3 spherical hinges are also arranged in a positive triangle.

When the parallel tracking mechanism is in motion, the three rope lengths are respectively $l_{1}, l_{2}, l_{3}$, and the position positive solution equation of the parallel tracking mechanism is[7]:

According to the parallel sun tracking mechanism of positive solutions of the conditions, $l_{1}, l_{2}, l_{3}$ are known, so the three unknowns are corresponding three independent equations. Through solving formula, the parallel tracking mechanism output equations of position $\alpha, \beta, Z_{B}$ can be obtained.

$$
\left\{\begin{array}{l}
l_{1}^{2}=(r \cos \beta-R)^{2}+\left(z_{B}-r \sin \beta\right)^{2} \\
l_{2}^{2}=\left(\frac{R}{2}-\frac{r}{2} \cos \beta+\frac{\sqrt{3}}{2} r \sin \alpha \sin \beta\right)^{2}+\left(\frac{\sqrt{3}}{2} r \cos \alpha-\frac{\sqrt{3}}{2} R\right)^{2}+\left(\frac{r}{2} \sin \beta+\frac{\sqrt{3}}{2} r \sin \alpha \cos \beta+z_{B}\right)^{2} \\
l^{2}{ }_{3}=\left(\frac{R}{2}-\frac{r}{2} \cos \beta-\frac{\sqrt{3}}{2} r \sin \alpha \sin \beta\right)^{2}+\left(\frac{\sqrt{3}}{2} r \cos \alpha-\frac{\sqrt{3}}{2} R\right)^{2}+\left(\frac{r}{2} \sin \beta+\frac{\sqrt{3}}{2} r \sin \alpha \cos \beta-z_{B}\right)^{2}
\end{array}\right.
$$

\section{RESEARCH ON CONTROL SYSTEM OF NEW SOLAR TRACKING SYSTEM}

After completing the mathematical modeling of the solar automatic tracking mechanism, it is necessary to design the control system, including the design of the hardware system and software system. This paper is proposed a control strategy of combining the photoelectric tracking with sun trajectory tracking based on the solar automatic tracking in any weather condition. The tracking mode is mainly depended on the trajectory tracking and sun trajectory tracking is supplemented. In a sunny day, a photosensitive diode is used to accomplish the light intensity detection. Cloudy or cloudy time is calculated by software to calculate the trajectory of the sun. Collaborative control of hardware and software enables real-time and accurate tracking of the sun. The working principle of the control system is shown in Fig. 2.

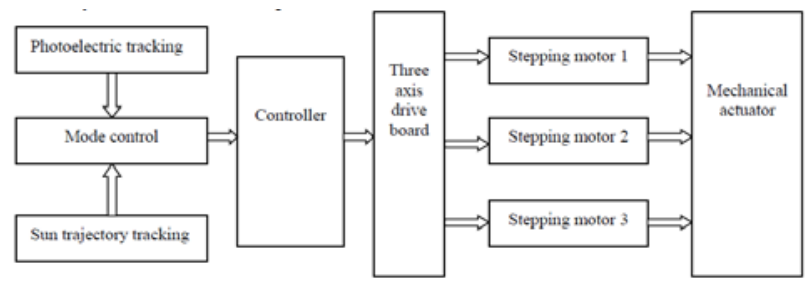

FIGURE II. THE WORKING PRINCIPLE OF THE NEW SOLAR AUTOMATIC TRACKING CONTROL SYSTEM

The new principle of automatic solar tracking control system is that the three ropes length displacement are calculated according to the kinematics equation of the 3-DOF parallel mechanism, then the controller will transmit control signals to the three shafts drive board, so the stepper motor can drive actuator motion. When the sun shines on the photoelectric sensor, the sensor is judged according to the information of the sunlight to see whether the threshold of the photoelectric sensor is set. If the threshold is set, the system is automatically switched from mode control to photoelectric tracking module. If the threshold of the sensor is not reached, the system will automatically switch to sun trajectory tracking module. According to the local latitude, the solar altitude angle and azimuth angle are calculated on the basis of the sun trajectory algorithm, and then the database is set up to store the information of the solar trajectory into the controller. When cloudy or dark clouds, the solar altitude angle and azimuth information are queried from the database in the current time., at and then the telescopic rope length is calculated by the controller. It can control the stepper motor movement through the program and make the solar panel reach the expected position. The combination of photoelectric tracking and sun trajectory tracking, the system can accurately track the solar rays all day and automatically.

\section{A. The Hardware Circuit Design of Control System}

The hardware of the automatic tracking control system is mainly composed of MCU controller, stepping motor, motor drive, photoelectric sensor, light intensity detection circuit and so on. Among them, the MCU is the control component, the stepping motor is the executive part, and the photoelectric sensor is the feedback control component. The hardware design of the control system is shown in Fig. 3. 


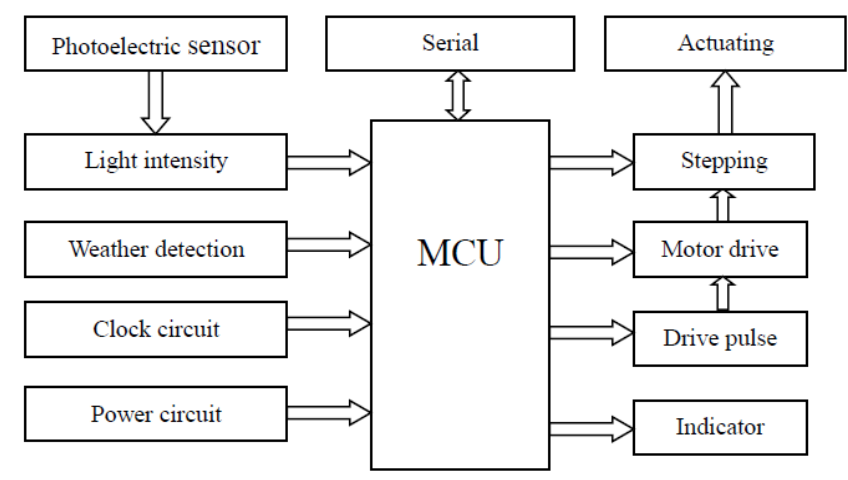

FIGURE III. THE HARDWARE DESIGN BLOCK DIAGRAM OF CONTROL SYSTEM

The control system constructs the application system based on MCU controller as the core. The modular thought of hardware circuit is designed for each unit circuit, and then the synthesis of the hardware circuit is carried out. Each circuit unit is combined to a complete hardware circuit by the function demands. The analog and digital signals are acquired through the peripheral circuit of the MCU control system, and input to the MCU control. After dealing with the MCU, the corresponding action of execution unit can control the whole system.

\section{B. The Software Design of Control System}

The software design of the automatic tracking system mainly includes the main control module, the photoelectric tracking module, the sun trajectory tracking module and the maximum power point tracking algorithm program design. The design of control system software adopts the idea of modular. All modules were designed and then combined to a complete module, so it is easy to program, but also can be easily modify error in a module. The overall design is shown in Fig. 4.

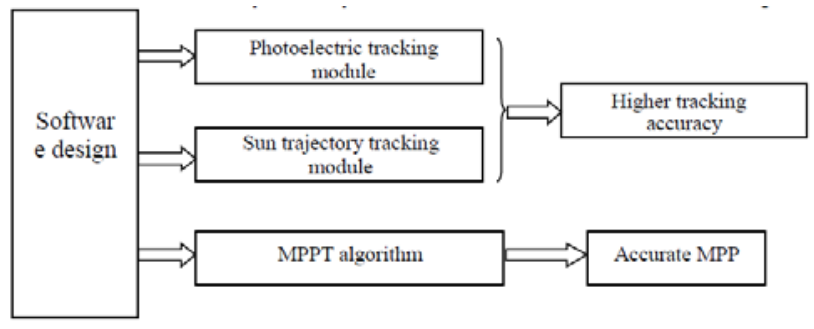

FIGURE IV. THE SOFTWARE DESIGN BLOCK DIAGRAM OF CONTROL SYSTEM

The program is composed of photoelectric tracking module and sun trajectory tracking module. Firstly, the photoelectric signal is converted into an electric signal through the light intensity detecting circuit according to the photoelectric sensor. According to the comparison of the preset reference voltage and the ends sensor voltage, if the ends sensor voltage is greater than the reference value, it can output high level. The stepper motor control will be not moved at this time. If it is less than the reference value, it can output low level. Then, the stepper motor control will be action by the MCU. Secondly, the solar altitude and azimuth are calculated according to the local latitude and the time. The angle of calculation will be built the data base, and then the solar elevation and azimuth are inquired every time from the library table. The MCU will send a pulse signal and direction signal to the stepper motor. The solar panel is corrected by one direction so as to complete a tracking. The solar panel is corrected by one direction so as to complete a tracking.

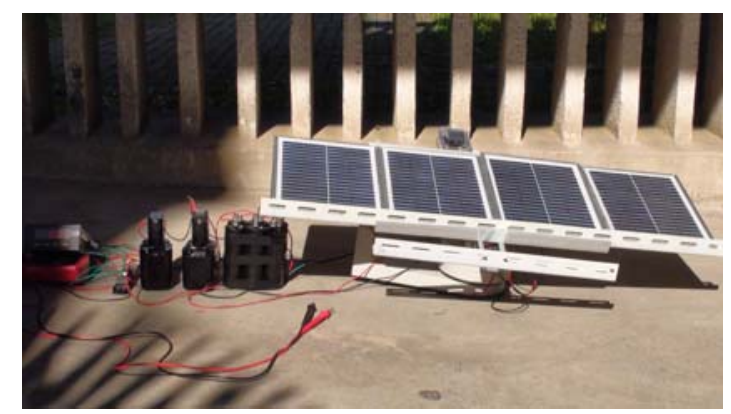

FIGURE V. THE POWER TEST PROCESS OF TWO AXIS TRACKING

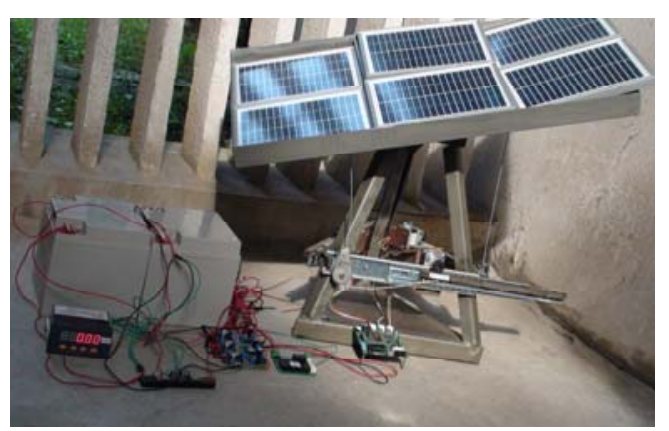

FIGURE VI. THE POWER TEST PROCESS OF NEW AUTOMATIC TRACKING SYSTEM

\section{EXPERIMENTAL RESEARCH AND ANALYSIS}

The test starts at 8:00 am and ends at 18:00 pm with a total of 10 hours of testing. Compared to the power of the two tracks, the result is shown in Fig. 7. In this experiment, one area of Jinhua as an example, the same power of solar panels (20W) is used in this test. The power comparison test is carried on the new solar automatic tracking system and two axis tracking system in different time on the same day. Test conditions: temperature $15-28{ }^{\circ} \mathrm{C}$, cloudless weather. The experiments are shown in Fig. 5 and Fig. 6.

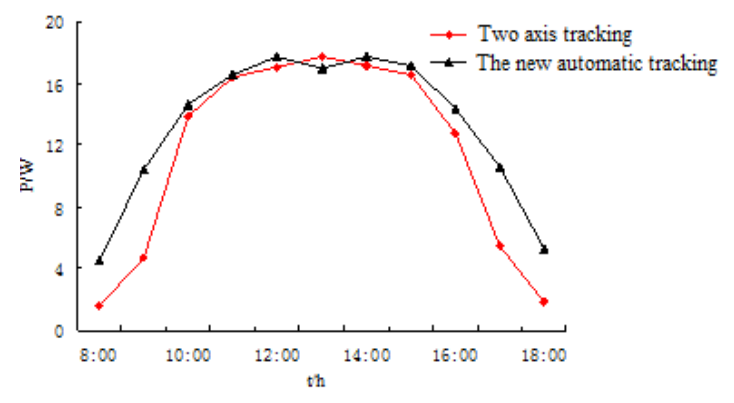

FIGURE VII. COMPARISON OF POWER GENERATION BETWEEN NEW AUTOMATIC TRACKING AND TWO AXIS TRACKING 
Fig.7 shows that the average power of the two axes tracking is about $11.6 \mathrm{~W}$ all day long. The average power of the new solar automatic tracking is about $13.5 \mathrm{~W}$ all day long. The new automatic tracking power is $14.1 \%$ higher than the traditional two axes tracking all day long. The experimental results show that the power generation of the new solar automatic tracking is better than the two axes tracking.

\section{V.CONCLUSIONS}

1. The designed new solar automatic tracking system can coordinate movement with each other without interference when three motors turn .

2. The whole power generation of the new solar automatic tracking system is $14.1 \%$ more than traditional two axis tracking system.

Compared with the traditional two axis tracking system, the designed new parallel solar tracking system is effective in generating more power in this paper. Its economy is obvious, and the utilization of the sun is high.

\section{ACKNOWLEDGEMENT}

This research was financially supported by the National Science Foundation.

\section{REFERENCE}

[1] Ardehali M M, Shahrestani M, Charles C. Energy simulation of solar assisted absorption system and examination of clearness index effects on auxiliary heating[J]. Energy Conversion and Management, 2007, 48(3): 864-870.

[2] CHEN Jianbin, SHEN Huiping, DING Lei, et al. Newest progresses on the two-axis tracking system study of the solar energy photovoltaic [J]. Machinery Design \& Manufacture, 2010(8): 364-266.

[3] YOU Jinzheng, ZOU Lixin, ZHOU Tong et al. Design of Automatic Solar Tracking System[J]. 2009,19(5):139-142.

[4] ZHANG Shunxin, SONG Kaifeng, FAN Shuncheng, et al. Study on the new two-axes solar tracker based on spherical parallel mechanism[J]. Journal of Hebei university of technology, 2003, 32(6):44-48.

[5] Sokolov A, Xirouchakis P. Dynamics analysis of a 3-DOF parallel manipulator with R-P-S joint structure[J]. Mechanism and Machine Theory, 2007, 42 (5): 541-557.

[6] LU Kaijiang, NIU Lufeng, LIU Yaru, et al. Research on Singular Configuration and Workspace of 3-RPS Parallel Mechanism[J]. Journal of agricultural machinery, 2007, 38(5):143-146.

[7] HE Xinsheng, GAO Chunfu, WANG Bin, et al. Positional Posture Analysis and Experimental Research on A New Sun Auto-tracking Mechanism[J]. Opt. Precision Eng., 2012, 20(5): 1048-1054 